\section{Short-segment jejunal carcinoma mimicking multiple lesions of the small bowel at capsule endoscopy}

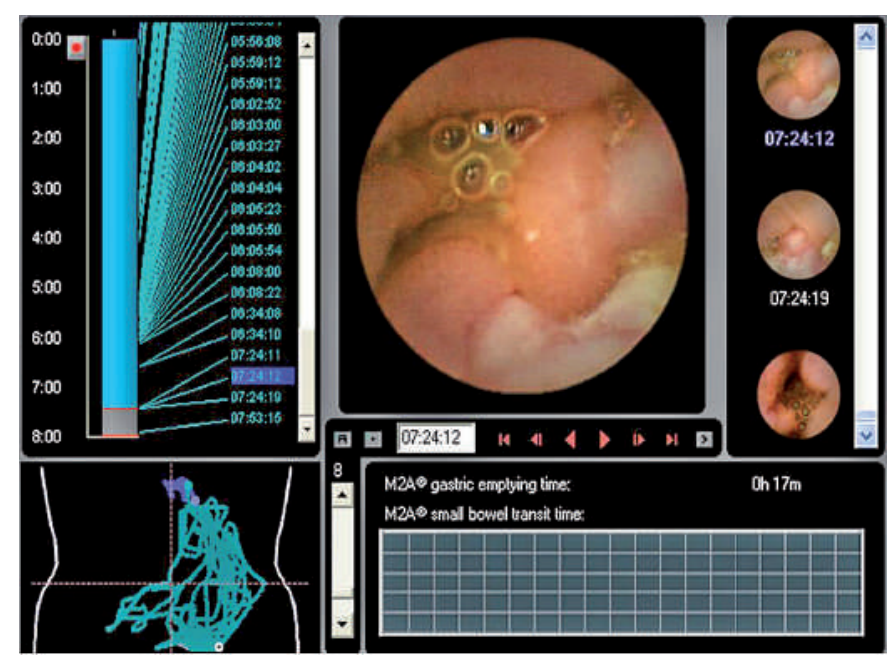

Figure 1 Capsule endoscopy images apparently showing several ulcerated stenotic areas at different locations in the intestine over a period of several hours.
Wireless capsule endoscopy is the procedure of choice for the assessment of patients with gastrointestinal bleeding of obscure origin [1,2]. We describe a patient with obscure gastrointestinal bleeding, in whom multiple lesions detected at capsule endoscopy were later found to be a single, short-segment neoplastic lesion.

A 54-year-old man presented with a history of several episodes of melena, occurring over the preceding year. Repeated radiological and endoscopic examinations were negative. Abdominal computed tomography (CT) showed an exophytic mass $(3 \times 4 \mathrm{~cm})$ in the proximal jejunum. Capsule endoscopy (Figure $\mathbf{1}$ ) and push enteroscopy revealed a jejunal stenosis. The surgical specimen is shown in Figure 2.

The clinical picture in patients with small-bowel disease may be misleading, even after thorough evaluation, and pathological abnormalities are difficult to diagnose, even when several advanced diagnostic tools are employed. In this patient, both small-bowel enema and CT scans with bowel opacification failed to identify the stenosis, which, probably due to its short length, had not caused proximal bowel dilatation. The enlarged lymph node (visualized on CT as adhering to the gut wall) was thought to represent an extraluminal lesion.
Because the video capsule was probably pushed backward several times as a result of retrograde movement, the recorded images yielded a false impression of progression through multiple stenotic tracts, suggesting the presence of metachronous lesions [3], until it forced its way through and progressed distally.

However, the identification of the abnormality in the proximal jejunum allowed us to reach it by push enteroscopy, and to obtain tissue samples. Because primary adenocarcinoma of the small bowel may be amenable to surgical treatment, provided it is at an early stage disease, with minimal lymph node involvement [4], we feel that the approach we adopted may have greatly improved the chance of survival for this patient.

Endoscopy_UCTN_Code_CCL_1AC_2AC

\section{G. Bassotti ${ }^{1}$, D. Castellani ${ }^{1}$,}

M. C. Bellucci ${ }^{2}$, P. Giovenali ${ }^{3}$, G. Natalini ${ }^{4}$, A. Morelli'

${ }^{1}$ Gastroenterology and Hepatology Section, University of Perugia Medical School, Perugia, Italy

${ }^{2}$ Radiology Section, University of Perugia

Medical School, Perugia, Italy

3 Pathology Section, "R. Silvestrini"

Hospital, Perugia, Italy

${ }^{4}$ General Surgery Section, “R. Silvestrini” Hospital, Perugia, Italy.

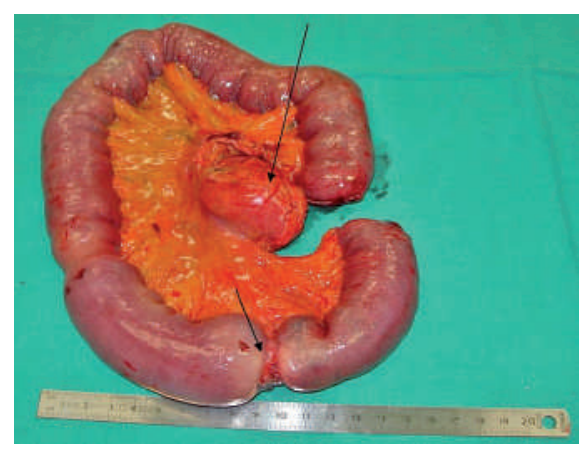

Figure 2 Laparotomy revealed a 1-cm jejunal stenosis (short arrow), without any associated bowel dilatation, and a 3-cm-diameter mesenteric lymph node (long arrow). Histological examination revealed the tumor to be a well-differentiated adenocarcinoma.

\section{References}

${ }^{1}$ Fleischer D. Capsule imaging. Clin Gastroenterol Hepatol 2005; 3 (Suppl 1): S30-S32

${ }^{2}$ Davis BR, Harris H, Vitale GC. The evolution of endoscopy: wireless capsule cameras for the diagnosis of occult gastrointestinal bleeding and inflammatory bowel disease. Surg Innov 2005; 12: 129- 133

${ }^{3}$ Varghese R, Weedon R. "Metachronous" adenocarcinoma of the small intestine. Int J Clin Pract 2005; 59 (Suppl 147): 106-108

${ }^{4}$ Dabaja BS, Suki D, Pro B et al. Adenocarcinoma of the small bowel: presentation, prognostic factors, and outcome of 217 patients. Cancer 2004; 101: 518-526

\section{Corresponding author}

\section{G. Bassotti, M.D.}

Clinica di Gastroenterologia ed Epatologia Via Enrico Dal Pozzo Padiglione W, 06100 Perugia, Italy

Fax: +39-075-584-7570

Email: gabassot@tin.it 